\title{
STOCHASTIC APPROACH VERSUS MULTIOBJECTIVE APPROACH FOR OBTAINING EFFICIENT SOLUTIONS IN STOCHASTIC MULTIOBJECTIVE PROGRAMMING PROBLEMS
}

\author{
Rafael Caballero $^{(1)}$, Emilio Cerdá ${ }^{(2)}$, María del Mar Muñoz ${ }^{(1)}$ and Lourdes Rey ${ }^{(1)}$
}

\begin{abstract}
In this work, we deal with obtaining efficient solutions for stochastic multiobjective programming problems. In general, these solutions are obtained in two stages: in one of them, the stochastic problem is transformed into its equivalent deterministic problem, and in the other one, some of the existing generating techniques in multiobjective programming are applied to obtain efficient solutions, which involves transforming the multiobjective problem into a problem with only one objective function. Our aim is to determine whether the order in which these two transformations are carried out influences, in any way, the efficient solution obtained. Our results show that depending on the type of stochastic criterion followed and the statistical characteristics of the initial problem, the order can have an influence on the final set of efficient solutions obtained for a given problem.
\end{abstract}

Keywords: Stochastic Multiobjective Programming, Efficiency, Stochastic Approach, Multiobjective Approach.

(1) Department of Applied Economics (Mathematics), University of Málaga, Málaga, Spain.

(2) Department of Foundations of Economic Analysis, University Complutense of Madrid, 28223 Madrid, Spain. 


\section{Introduction}

In the field of Operations Research (OR) we often encounter real problems in which the Decision Maker (DM) wishes to optimize several objectives at the same time and, moreover does not know the values of some parameters at the moment he or she has to make the decision. When these unknown parameters can be considered as random variables, the resulting problem is denominated in OR Stochastic Multiobjective Programming Problem.

The achievement of efficient solutions in multiobjective programmnig problems in which some parameters are random variables has hitherto been analysed from different points of view. This question is not easy to approach, because the fact that the objectives of the problem depend on random variables makes these objectives random variables also. Given that, in general, a random variable does not admit an order relation, a solution may be efficient in the Pareto sense of a multiobjective stochastic problem for obtaining a specific value for the random variables which intervene in the problem and not for others.

Therefore, in order to obtain the efficient solutions for these problems we need to define previously the specific concepts of efficient solution that will be used. In general, the studies approach the resolution of such problems with techniques from stochastic programming and multiobjective programming. Thus, Stancu-Minasian (1984) points out that the resolution of such problems always involves a double transformation: transforming the multiobjective problem into a problem with only one objective function and the stochastic problem into its equivalent deterministic problem. The same steps are also considered by Ben Abdelaziz (1992) who also classifies the existing techniques available for the resolution of these problems according to the order in which the transformations are carried out. In this classification, Ben Abdelaziz uses the term multiobjective approach to refer to the set of techniques that solve the problem by considering first the transformation of the stochastic multiobjective problem into its equivalent multiobjective deterministic problem; and the term stochastic approach to refer to those techniques that transform the stochastic multiobjective problem into a stochastic problem with only one objective function to be solved later with any of the existing techniques 
in the literature. The transformations which are carried out in both approaches appear in figures 1 and 2 .

STOCHASTIC
MULTIOBJECTIVE
PROGRAMMING
PROBLEM

\begin{tabular}{|c|}
\hline EQUIVALENT \\
DETERMINISTIC \\
MULTIOBJECTIVE \\
PROBLEM \\
\hline
\end{tabular}

EQUIVALENT

DETERMINISTIC

MONOBJECTIVE

PROBLEM 
FIGURE 1. STEPS FOR OBTAINING EFFICIENT SOLUTIONS IN THE MULTIOBJECTIVE APPROACH.

STOCHASTIC
MULTIOBJECTIVE
PROGRAMMING
PROBLEM

\begin{tabular}{|c|}
\hline STOCHASTIC \\
MONOBJECTIVE \\
PROGRAMMING \\
PROBLEM \\
\hline
\end{tabular}

EQUIVALENT DETERMINISTIC MONOBJECTIVE PROBLEM 


\section{FIGURE 2. STEPS FOR OBTAINING EFFICIENT SOLUTIONS IN THE STOCHASTIC APPROACH.}

Ben Abdelaziz also points out that both approaches present shortcomings because they emphasise either the multiobjective or the stochastic nature of the problem to the detriment of the other, and so the stochastic and multiobjective nature of the problem are not simultaneously considered.

In this paper, we focus on obtaining efficient solutions for problems with continuous random variables influencing only the objective functions. That is, we consider that the feasible set of the problem is deterministic or that it has been transformed into its equivalent deterministic, requiring that the constraints hold with, at lest, a given probability (this question is largely analysed in different works such us Kall and Wallace (1994), Prékopa (1997) or Lin and Iwamura (1997)). Moreover, we focus on problems in which the random variables appearing in the problem are continuous. The analysis of the efficiency in multiobjective stochastic programming when the random variables are discrete has been studied in different papers among which we can highlight Ben Abdelaziz (1992) and Ben Abdelaziz, Lang and Nadeau $(1997,1999)$.

For these problems we have to point out that, so far, different concept of efficient solution has been defined. These definitions fit within what Ben Abdelaziz (1992) calls the multiobjective approach and are determined by selecting a criterion that will be used to transform each stochastic objective into its equivalent deterministic. Then, an equivalent deterministic multiobjective problem is formulated, which has a corresponding set of efficient solutions, which, in turn, is considered the efficient set of the initial problem. In this way, we find in the literature the concepts of expected value efficient solution, minimum variance efficient solution, or expected value standard deviation efficient solution (see, for example, White (1982)). Other concepts defined in this way are those of minimum risk efficiency with aspiration levels $u_{1}, \ldots, u_{q}$ defined by Stancu-Minasian and Tigan (1984) or those of efficiency in probability of Goicoechea, Hansen and Duckstein (1982).

Taking these concepts into account, and the criticism regarding these approaches, we may ask: if, in the stochastic approach, we use any of the available multiobjective programming 
techniques to obtain efficient solutions for the stochastic multiobjective problem and the resulting stochastic problem is later solved by some of the following criteria - expected value, minimum variance, minimum risk, or Kataoka's criterion - would the optimal solution for the resulting problem be in any way related to the concepts of efficiency outlined by the multiobjective approach?

In this work, we deal with this issue. In the stochastic approach we consider the application of the weighting method to the initial problem, because this is one of the more widely used generating techniques in deterministic multiobjective programming. We have organised our study according to the kind of stochastic criterion applied to obtain the equivalent deterministic problem. For each criterion, we compare the optimal solutions for the weighted problem, corresponding to the stochastic approach, to the efficient solutions obtained with the multiobjective approach. First, we will look into some of the results of multiobjective programming that will be dealt with in our work.

\section{Formulation of the Problem and Efficient Solution Concepts}

Let us consider the following stochastic multiobjective problem:

$$
" \operatorname{Min}_{\mathbf{x} \in D} \tilde{\mathbf{z}}(\mathbf{x}, \tilde{\mathbf{c}})=\left(\tilde{z}_{1}\left(\mathbf{x}, \tilde{\mathbf{c}}_{)}, \tilde{z}_{2}(\mathbf{x}, \tilde{\mathbf{c}}), \ldots, \tilde{z}_{q}(\mathbf{x}, \tilde{\mathbf{c}})\right)\right.
$$

In this problem the objective functions of the problem depend on a vector of continuous random parameters, $\tilde{\mathbf{c}}$, defined over a set $E \subset R^{s}$. Let us assume a family of events $F$, i.e., subsets of $E$, and the probability distribution $P$ defined on $F$, so that for any subset of $\mathrm{E}, A \subset \mathrm{E}, A \in \mathrm{F}$, the probability of $A, P(A)$, is known. Also, we maintain the hypothesis that the probability distribution, $P$, is independent from the decision variables of the problem, $x_{1}, \ldots, x_{n}$. We also assume that the feasible set of the problem, $D \subset \mathrm{R}^{\mathrm{n}}$, is convex and, moreover, is deterministic or has been transformed into its equivalent deterministic using the chance constrained method.

As has been previously pointed out, obtaining efficient solutions for this problem has been hitherto considered from two approaches: the multiobjective approach and the stochastic approach, that we subsequently analyse. 


\subsection{Multiobjective Approach}

In this approach, the stochastic objectives are transformed into their equivalents deterministic in accordance with any of the criteria of stochastic programming, obtaining an equivalent deterministic multiobjective problem which has a set of efficient solutions which are considered efficient for the original problem. We can define, from this idea, the following concept of efficient solution for problem (SMP):

\section{Definition 1: Expected value efficient solution (White (1982))}

$\mathbf{x} \in D$ is an expected value efficient solution to the problem (SMP) if it is Pareto efficient to the problem:

$$
\operatorname{Min}_{\mathbf{x} \in D}\left(\bar{z}_{1}(\mathbf{x}), \bar{z}_{2}(\mathbf{x}), \ldots, \bar{z}_{q}(\mathbf{x})\right)
$$

where $\bar{z}_{k}(\mathbf{x})$ is the expected value of the random variable $\tilde{z}_{k}(\mathbf{x}, \tilde{\mathbf{c}}), k \in\{1,2, \ldots, q\}$.

That is, given the problem (SMP), we apply the expected value criterion to each one of the stochastic objective functions of the problem and we obtain the equivalent deterministic multiobjective problem (E).

Therefore, with this criterion each stochastic objective is substituted by the expected value of each stochastic objective which is a measure of central trend.

Let us now consider the application of the minimum variance criterion to transform the stochastic multiobjective problem into its equivalent deterministic problem. As the variance of a random variable is defined as the expected value of the square of the deviation of the random variable around its expected value, the choice of this criterion implies the choice of the vector $\mathrm{x}$ for which the random variable $\tilde{z}(\mathbf{x}, \tilde{\mathbf{c}})$ is most concentrated around its expected value. Therefore, the equivalent deterministic minimum variance can be interpreted as a measure of quadratic error.

In the multiobjective approach, the application of this criterion gives the following concept: 
Definition 2: Minimum variance efficient solution (White (1982))

$\mathbf{x} \in D$ is a minimum variance efficient solution to the stochastic multiobjective programming problem (SMP) if it is a Pareto efficient solution to the problem:

$$
\operatorname{Min}_{\mathbf{x} \in D}\left(\boldsymbol{\sigma}_{1}^{2}(\mathbf{x}), \boldsymbol{\sigma}_{2}^{2}(\mathbf{x}), \ldots, \boldsymbol{\sigma}_{q}^{2}(\mathbf{x})\right)
$$

where $\sigma_{k}^{2}(\mathbf{x})$ is the variance of the $k$-th objective function, $k \in\{1,2, \ldots, q\}$.

Let $\mathcal{E}_{\sigma^{2}}$ be the set of efficient solutions to problem (V).

\section{Definition 3: Expected value standard deviation efficient solution}

$\mathbf{x} \in D$ is an expected value standard deviation efficient solution to the stochastic multiobjective programming problem (SMP) if it is a Pareto efficient solution to the problem:

$$
\operatorname{Min}_{\mathbf{x} \in D}\left(\bar{z}_{l}(\mathbf{x}), \ldots, \bar{z}_{q}(\mathbf{x}), \boldsymbol{\sigma}_{l}(\mathbf{x}), \ldots, \boldsymbol{\sigma}_{q}(\mathbf{x})\right)
$$

which includes the expected value and the standard deviation of the problem's stochastic objective functions.

Let $\mathcal{E}_{E \sigma}$ be the set of expected value standard deviation efficient solutions to problem (SMP).

Let us observe that through this concept of efficiency a measure of central trend and a measure of dispersion of each stochastic objective are registered. This criterion of solution of problems of stochastic programming has been largely used in portfolio models in Finance Economics in order to solve stochastic programming problems corresponding to economic models.

We will now deal with the application of maximum probability — the minimum risk and Kataoka's criteria - to stochastic multiobjective programming problems.

Given the problem (SMP), we can apply the minimum risk criterion to each stochastic objective separately. In this case, the decision maker (DM) must fix a priori an aspiration level, $u_{k}$, for each stochastic objective function and find the vector $\mathbf{x}$, in which the probability 
of the $k$-th objective function not being greater than the aspiration level fixed is maximum: $P\left(\tilde{z}_{k}(\mathbf{x}, \tilde{\mathbf{c}}) \leq u_{k}\right)$. The separate application of this criterion to each stochastic objective function in the problem (SMP) lead us to the following definition of efficiency:

Definition 4: Minimum risk efficient solution for levels $u_{1}, \ldots, u_{q}$ (Stancu-Minasian and Tigan (1984))

$\mathbf{x} \in D$ is a vectorial minimum risk solution for levels $u_{1}, u_{2}, \ldots, u_{q}$ if it is a Pareto efficient solution to the problem:

$$
\operatorname{Max}_{\mathbf{x} \in D}\left(P\left(\tilde{z}_{1}(\mathbf{x}, \tilde{\mathbf{c}}) \leq u_{1}\right), \ldots, P\left(\tilde{z}_{q}(\mathbf{x}, \tilde{\mathbf{c}}) \leq u_{q}\right)\right)
$$

$\mathcal{E}_{M R}(\mathbf{u})$ denotes the set of efficient solutions to problem $(\mathrm{MR}(\mathbf{u}))$.

The second criterion of maximum probability is the Kataoka's criterion. In this case, we fix a probability for the stochastic objective function, $\beta_{k}$, and look for the smaller value $u_{k}$ for which we can assert that, given the fixed probability, the stochastic objective function does not exceed the level. From the application of this criterion to each stochastic objective function in the problem (SMP), we can define the following concept of efficiency:

Definition 5: Efficient solution with probabilities $\boldsymbol{\beta}_{1}, \boldsymbol{\beta}_{2}, \ldots, \boldsymbol{\beta}_{q}$ or $\boldsymbol{\beta}$-Efficient solution Let $\mathbf{x} \in D ; \mathrm{x}$ is an efficient solution with probabilities $\boldsymbol{\beta}_{1}, \boldsymbol{\beta}_{2}, \ldots, \boldsymbol{\beta}_{q}$ if there exists any $\mathbf{u} \in \mathrm{R}^{\mathrm{q}}$ such that $\left(\mathbf{x}^{\mathbf{t}}, \mathbf{u}^{\mathbf{t}}\right)^{\mathbf{t}}$ is an efficient solution to problem

$$
\begin{array}{ll}
\operatorname{Min}_{\mathbf{x}, \mathbf{u}} & \left(u_{1}, \ldots, u_{q}\right) \\
\text { s.t } & P\left(\tilde{z}_{k}(\mathbf{x}, \tilde{\mathbf{c}}) \leq u_{k}\right) \geq \boldsymbol{\beta}_{k}, k=1,2, \ldots, q \\
& \mathbf{x} \in D
\end{array}
$$

The set of efficient solutions with probabilities $\boldsymbol{\beta}_{1}, \boldsymbol{\beta}_{2}, \ldots, \boldsymbol{\beta}_{q}$ for the problem (SMP) is denoted by $\mathcal{E}_{k}(\boldsymbol{\beta}) \subset \mathrm{R}^{\mathrm{n}}$.

This concept is no more than a generalisation of the concept introduced by Goicoechea, Hansen and Duckstein (1982), who set the same probability for all the stochastic objective functions; that is, these authors consider $\boldsymbol{\beta}_{k}=\boldsymbol{\beta}$, for every $k \in\{1,2, \ldots, q\}$. 
The two efficiency concepts of maximum probability we have just defined enable us to obtain an efficient set for each vector of aspiration levels or probabilities. Further, the distribution functions of the stochastic objective functions are involved in both concepts. Caballero, Cerdá, Muñoz, and Rey (2000) relate both of these concepts as we will show in the following theorem.

\section{Theorem 2 (Caballero, Cerdá, Muñoz, and Rey (2000))}

Let us assume that the distribution function of the random variable $\tilde{z}_{k}(\mathbf{x}, \tilde{\mathbf{c}})$ is continuous and strictly increasing. Then, $\mathbf{x}$ is an efficient solution to problem $(\operatorname{MR}(\mathbf{u}))$ if and only if $\left(\mathbf{x}^{\mathbf{t}}, \mathbf{u}^{\mathbf{t}}\right)^{\mathrm{t}}$ is an efficient solution to problem $(K(\boldsymbol{\beta}))$, with $\mathbf{u}$ and $\boldsymbol{\beta}$ such that:

$$
P\left(\tilde{z}_{k}(\mathbf{x}, \tilde{\mathbf{c}}) \leq u_{k}\right)=\beta_{k}, \quad \forall k \in\{1,2, \ldots, q\}
$$

Corollary 1 (Caballero, Cerdá, Muñoz, and Rey (2000))

$$
\bigcup_{\mathbf{u} \in \mathrm{R}^{\mathrm{q}}} \mathcal{E}_{\mathrm{MR}}(\mathbf{u})=\bigcup_{\boldsymbol{\beta} \in B} \mathcal{E}_{\mathrm{K}}(\boldsymbol{\beta})
$$

with $B=\left\{\boldsymbol{\beta} \in \mathrm{R}^{\mathrm{q}} / \beta_{\mathrm{k}} \in(0,1), k=1,2, \ldots, q\right\}$.

From the results obtained, we can see that the union of the sets of efficient points of both problems coincide. Moreover, if for some fixed probabilities $\boldsymbol{\beta}=\left(\boldsymbol{\beta}_{l}, \boldsymbol{\beta}_{2}, \ldots, \boldsymbol{\beta}_{q}\right)^{\mathrm{t}}, \mathbf{x} \in D$ is an efficient solution to problem $\mathrm{K}(\boldsymbol{\beta})$, we know from Theorem 2 that it is also a minimum risk efficient solution of levels $u_{1}, u_{2}, \ldots, u_{q}$ and vice versa. Further, the aspiration levels and the probabilities will have the relation established in Theorem 2. This result allows us to perform the analysis of these efficient solutions by either of the two concepts and, from Theorem 2, to obtain the level or the probability for which it is efficient in accordance with the other.

On the other hand, in Caballero, Cerdá, Muñoz, Rey, and Stancu-Minasian (2001), other relations among the five concepts of efficient solution defined in the multiobjective approach are established. The relations stated in the two papers referred to permit these efficient sets to be characterized and establish which efficient criterion is the most appropriate in a specific problem, using as a starting point the preferences of the DM. As each one of 
these criteria registers some statistical characteristics of the objectives, at first, the sets of the defined efficient solutions are different and apparently not related.

\subsection{Stochastic Approach}

Let us consider again the problem (SMP). In order to obtain efficient solutions to this problem it is also possible to apply first any of the techniques for obtaining efficient solutions used in multiobjective programming which leads, in general, to solve a stochastic programming problem with one objective function which has to be solved next, applying any of the criteria of solution to the stochastic programming. As has been previously pointed out, this order in the transformations has been called in the literature stochastic approach.

Among the existing methods in deterministic multiobjective programming, one of the most important in order to obtain efficient solutions is the weighting method. In this method, a non-negative weight is assigned to each one of the objective functions of the problem. The aim is to minimise the sum of the objective functions of problem (SMP), which is weighted by the weight assigned to it. Let $\boldsymbol{\mu}=\left(\boldsymbol{\mu}_{1}, \ldots, \boldsymbol{\mu}_{q}\right)^{\mathrm{t}}$ be the weight vector, $\boldsymbol{\mu}_{k} \in \mathrm{R}^{+}$. The problem associated with problem (SMP) by the weighting method is the following stochastic programming problem:

$$
\text { "Min" } \tilde{\mathbf{x} \in D}(\mathbf{x}, \tilde{\mathbf{c}})=\sum_{k=1}^{q} \boldsymbol{\mu}_{k} \tilde{z}_{k}(\mathbf{x}, \tilde{\mathbf{c}})
$$

In order to solve it, we can apply one of the existing criteria in stochastic programming, e.g., expected value, minimum variance, minimum risk, or Kataoka's criterion. The application of these criteria to the problem (S) would lead us to obtain four different equivalent deterministic problems whose optimal solutions are efficient solutions to problem (SMP). From the previous comments, it can be asked if these efficient solutions maintain some relationship with the efficient solutions previously defined in the multiobjective approach. That is, can a solution which is efficient according to any of these approaches be efficient according to other approach? And, in that case, which one of the two approaches is most appropriate in order to obtain efficient solutions? As we will see in the next section, the answers to these questions are complex although under certain hypothesis it is possible to answer them. 
Before studying these questions, we consider it convenient to point out which relationships exist between the efficient solutions of a deterministic multiobjective problem and the optimal solutions to the corresponding weighted problem.

Let us consider the following deterministic multiobjective problem:

$$
\operatorname{Min}_{\mathbf{x} \in D}\left(z_{1}(\mathbf{x}), \ldots, z_{q}(\mathbf{x})\right)
$$

and the problem associated with problem $(\mathrm{P})$ by the weighting method:

$$
\operatorname{Min}_{\mathbf{x} \in D} \sum_{k=1}^{q} \boldsymbol{\mu}_{k} z_{k}(\mathbf{x})
$$

where $\boldsymbol{\mu}=\left(\boldsymbol{\mu}_{1}, \ldots, \boldsymbol{\mu}_{q}\right)^{\mathrm{t}}$ is the weight vector, $\boldsymbol{\mu}_{k} \in \mathrm{R}^{+}$.

The following theorem establishes the relationship between the problem's solution $(\mathrm{P}(\boldsymbol{\mu}))$ and the efficient solutions to problem $(\mathrm{P})$.

Theorem 1. Sawaragi, Nakayama and Tanino (1985).

a) Let us assume that functions $z_{1}, \ldots, z_{q}$ are convex, and $D$ is a convex set. If $\mathbf{x}^{*}$ is a properly efficient solution for the problem $(\mathrm{P})$, there exists $\boldsymbol{\mu}_{1}, \ldots, \boldsymbol{\mu}_{q}, \boldsymbol{\mu}_{k}>0$ for every $k \in\{1,2, \ldots, q\}$, such that $\mathbf{x}^{*}$ is the solution to problem $(\mathrm{P}(\boldsymbol{\mu}))$.

b) If $\mathbf{x}^{*}$ is the solution to problem $(\mathrm{P}(\boldsymbol{\mu}))$, with $\boldsymbol{\mu}>\mathbf{0}$, then, $\mathbf{x}^{*}$ is a properly efficient solution to problem $(\mathrm{P})$.

c) If $\mathbf{x}^{*}$ is the only solution to problem $(\mathrm{P}(\boldsymbol{\mu}))$, with $\boldsymbol{\mu} \geq \mathbf{0}$, then $\mathbf{x}^{*}$ is an efficient solution to problem $(\mathrm{P})$. If this is not the only one, the solutions obtained for $(\mathrm{P})$ are weakly efficient.

From this theorem, if the convexity of the feasible set and the objective functions is verified, the set of properly efficient solutions to problem $(\mathrm{P})$ can be obtained by simply solving problem $(\mathrm{P}(\boldsymbol{\mu}))$ for all possible values of the vector $\boldsymbol{\mu}$ with strictly positive components. On 
the other hand, if the convexity condition is not verified, some efficient solutions to the problem (P) might not be obtained by this method. Furthermore, from point (c) of this theorem, if we take weight vectors with some null components - which means that the objective function associated with this component will not be taken into account when solving problem $(\mathrm{P}(\boldsymbol{\mu})))$ — we can assert that the solution obtained is efficient only if it is the only solution to $(\mathrm{P}(\boldsymbol{\mu}))$. In any other case, we can only assert that the solutions obtained are weakly efficient.

\section{Stochastic Approach versus Multiobjective Approach}

In order to answer the stated questions about the relationships between the efficient solutions of a stochastic multiobjective problem we solve the weighted problem (S) by means of the following criteria: expected value, minimum variance, minimum risk and Kataoka. In each case, we will compare the efficient solutions obtained using the stochastic approach with some of the efficient solution concepts defined in section 2.1, corresponding to the multiobjective approach.

\subsection{Expected Value Criterion}

Let the problem be (S) and let us consider the expected value criterion in order to solve it. Then we obtain the following equivalent deterministic problem:

$$
\operatorname{Min}_{\mathbf{x} \in D} \bar{f}(\mathbf{x})=\sum_{k=1}^{q} \boldsymbol{\mu}_{k} \bar{z}_{k}(\mathbf{x})
$$

where $\bar{f}(\mathbf{x})$ denotes the expected value of the random variable $\tilde{f}(\mathbf{x})=\sum_{k=1}^{q} \mu_{k} \tilde{z}_{k}(\mathbf{x})$.

Thus, if we apply the expected value criterion to problem (S), the resulting problem minimises a linear combination of the expected value of the original problem's stochastic objective functions, and the coefficients of such a linear combination are no more than the weights assigned to the stochastic objectives in the first stage of the problem resolution. In other words, the problem obtained is the same as solving the original stochastic multiobjective programming problem, transforming the problem into its equivalent deterministic problem by applying the expected value criterion to each one of the stochastic objective functions of 
(SMP) and then applying the weighting method in order to obtain the expected value efficient solutions.

Therefore, based on the concept of expected value efficient solution and the results of the deterministic multiobjective programming, given that the weights, $\boldsymbol{\mu}_{k}, k \in\{1,2, \ldots, q\}$, are not negative, for each vector, $\boldsymbol{\mu} \in \mathrm{R}^{\mathrm{q}+}$, the relationship between problem (E) and problem (SE) is the same as the relationship between any multiobjective problem and its associated weighted problem, which is expressed in Theorem 1 .

\subsection{Minimum Variance Criterion}

Let us now consider the application of the minimum variance criterion to the weighted problem. Given that the random variable $\tilde{f}(\mathbf{x}, \tilde{\mathbf{c}})=\sum_{k=1}^{q} \mu_{k} \tilde{z}_{k}(\mathbf{x}, \tilde{\mathbf{c}})$ is a linear function of the random variables $\tilde{z}_{1}(\mathbf{x}, \tilde{\mathbf{c}}), \tilde{z}_{2}(\mathbf{x}, \tilde{\mathbf{c}}), \ldots, \tilde{z}_{q}(\mathbf{x}, \tilde{\mathbf{c}})$, its variance depends on the variances of these random variables and their covariances (see, for example, Hogg and Craig (1989), p. 177.). Thus, we have:

$$
\sigma^{2}(\mathbf{x})=\sum_{k=1}^{q} \mu_{k}^{2} \sigma_{k}^{2}(\mathbf{x})+2 \sum_{\substack{k, s=1 \\ k<s}}^{q} \mu_{k} \mu_{s} \sigma_{k s}(\mathbf{x})
$$

where $\sigma_{k s}(\mathbf{x})$ denotes the covariance of the random variables $\tilde{z}_{k}(\mathbf{x}, \tilde{\mathbf{c}})$ and $\tilde{z}_{s}(\mathbf{x}, \tilde{\mathbf{c}})$ :

$$
\sigma_{k s}(\mathbf{x})=E\left\{\left(\tilde{z}_{k}(\mathbf{x}, \tilde{\mathbf{c}})-\bar{z}_{k}(\mathbf{x})\right)\left(\tilde{z}_{s}(\mathbf{x}, \tilde{\mathbf{c}})-\bar{z}_{s}(\mathbf{x})\right)\right\}
$$

Therefore, the variance of the function $\tilde{f}(\mathbf{x}, \tilde{\mathbf{c}})=\sum_{k=1}^{q} \mu_{k} \tilde{z}_{k}(\mathbf{x}, \tilde{\mathbf{c}})$ depends not only on the variances of functions $\tilde{z}_{1}(\mathbf{x}, \tilde{\mathbf{c}}), \tilde{z}_{2}(\mathbf{x}, \tilde{\mathbf{c}}), \ldots, \tilde{z}_{q}(\mathbf{x}, \tilde{\mathbf{c}})$, but also on their covariances. Thus, if we apply the minimum variance criterion to resolve the problem (S), we obtain the problem:

$$
\operatorname{Min}_{\mathbf{x} \in D} \sigma^{2}(\mathbf{x})=\sum_{k=1}^{q} \mu_{k}^{2} \sigma_{k}^{2}(\mathbf{x})+2 \sum_{\substack{k, s=1 \\ k<s}}^{q} \mu_{k} \mu_{s} \sigma_{k s}(\mathbf{x})
$$


We can see that the relationships between a weighted problem and the multiobjective equivalent deterministic problem are not so direct as in the previous case. In order to establish these relationships we differentiate between the case of the covariances of the stochastic objective functions all being zero and the case of some of them not being zero.

If the covariances of the objective functions are zero, that is, if it fulfils the following:

$$
\boldsymbol{\sigma}_{k s}(\mathbf{x})=0 \text { for every } k, s \in\{1,2, \ldots, q\} \text {, with } k \neq s \text { and for every } \mathbf{x} \in D
$$

then, the problem (SV), resulting from applying the minimum variance criterion to the weighted problem ( $\mathrm{S})$, is:

$$
\operatorname{Min}_{\mathbf{x} \in D} \sigma^{2}(\mathbf{x})=\sum_{k=1}^{q} \mu_{k}^{2} \sigma_{k}^{2}(\mathbf{x})
$$

that is, the problem obtained is the same as the one we would have obtained by solving the stochastic multiobjective programming problem using the multiobjective approach, i.e., applying the minimum variance criterion to each stochastic objective function and then, the weighted method to the equivalent deterministic multiobjective problem. Both problems are related as shown by Theorem 1 .

The second situation to deal with is the case of the covariances of some stochastic objective functions are non-null. In this case, for each vector $\boldsymbol{\mu} \in \mathrm{R}^{\mathrm{q+}}$, the optimal solution to the problem (SV) does not have to be necessarily minimum variance efficient, as shown in section 4, where a linear stochastic bi-objective problem is formulated with stochastic objective functions whose covariance is not zero. In this example, we see that if we apply the minimum variance criterion to the weighted problem (S), the solution obtained is not necessarily minimum variance efficient. The only way to ensure efficiency is if the covariances of the objective functions are zero. As we know, the covariance of two random variables measures the dependence between them. In this sense, we know that if two random variables are independent, their covariance is zero. However, and generally speaking, the opposite is not necessarily true. In other words, the fact that the covariance is zero does not necessary means that the random variables are independent. Therefore, the application of the minimum 
variance criterion to the weighted problem reflects to some extent the dependency between the stochastic objective functions.

\subsection{Maximum Probability Criteria}

Let us now consider applying the criteria of maximum probability (minimum risk and Kataoka's) to the weighted problem:

$$
\text { "Min" } \tilde{\mathbf{x} \in D}(\mathbf{x}, \tilde{\mathbf{c}})=\sum_{k=1}^{q} \boldsymbol{\mu}_{k} \tilde{z}_{k}(\mathbf{x}, \tilde{\mathbf{c}})
$$

In order to apply the minimum risk criterion to this problem, we must fix a value $u$ (aspiration level of the problem's objective function) and maximise the probability of the random variable $\tilde{f}(\mathbf{x}, \tilde{\mathbf{c}})=\sum_{k=1}^{q} \mu_{k} \tilde{z}_{k}(\mathbf{x}, \tilde{\mathbf{c}})$ not exceeding such a value. The equivalent deterministic problem generated is:

$$
\operatorname{Max}_{\mathbf{x} \in D} P\left\{\sum_{k=1}^{q} \mu_{k} \tilde{z}_{k}(\mathbf{x}, \tilde{\mathbf{c}}) \leq u\right\}
$$

Given that $\tilde{f}(\mathbf{x}, \tilde{\mathbf{c}})=\sum_{k=1}^{q} \boldsymbol{\mu}_{k} \tilde{z}_{k}(\mathbf{x}, \widetilde{\mathbf{c}})$ is a function of the $q$ objective functions for the problem, the choice made for value $u$ in the previous problem is not a trivial issue. Note that this level must be fixed for the random variable $\tilde{f}(\mathbf{x}, \tilde{\mathbf{c}})=\sum_{k=1}^{q} \mu_{k} \tilde{z}_{k}(\mathbf{x}, \tilde{\mathbf{c}})$, where this variable is not an objective of the initial stochastic multiobjective programming problem, but has been constructed from it in order to resolve it. Therefore, value $u$, which in stochastic programming is the aspiration level fixed by the DM for the stochastic objective, no longer has this meaning. This could lead to think that the minimum risk criterion is not applicable to the weighted problem. However, we can decide to determine the value $u$ from an aspiration level for each objective. In such a case, the DM has to determine an aspiration level, $u_{k}$, for each objective function, and level $u$ is calculated as the addition of the levels fixed by the DM, weighting each level with the weight assigned to the corresponding objective. That is, $u=\sum_{k=1}^{q} \boldsymbol{\mu}_{k} u_{k}=\boldsymbol{\mu}^{\mathbf{t}} \mathbf{u}$. In this way, level $u$ is set to be a function of the aspiration levels fixed by the DM for each objective function, but weighted according to the significance given to 
each of them by the weight assigned to each objective. The equivalent deterministic problem is as follows:

$$
\operatorname{Max}_{\mathbf{x} \in D} P\left(\sum_{k=1}^{q} \mu_{k} \tilde{z}_{k}(\mathbf{x}, \tilde{\mathbf{c}}) \leq \sum_{k=1}^{q} \mu_{k} u_{k}\right)
$$

On the other hand, if we apply Kataoka's criterion to the weighted problem (S), the equivalent deterministic problem generated is as follows:

$$
\begin{gathered}
\operatorname{Min}_{\mathbf{x}, \mathrm{u}} u \\
\text { s.t } P\left(\sum_{k=1}^{q} \boldsymbol{\mu}_{k} \tilde{z}_{k}(\mathbf{x}, \tilde{\mathbf{c}}) \leq u\right)=\boldsymbol{\beta} \\
\mathbf{x} \in D
\end{gathered}
$$

where $\beta$ is the probability fixed for the problem. The resolution of this problem determines the lowest $u$ level the objective function of the weighted problem with probability $\beta$ can reach. Once again, the question arises whether it is advisable or not to apply this criterion to solve the initial stochastic multiobjective programming problem, because applying this criterion on the weighted problem (S) involves the need to fix a probability for the random variable $\tilde{f}(\mathbf{x}, \tilde{\mathbf{c}})=\sum_{k=1}^{q} \mu_{k} \tilde{z}_{k}(\mathbf{x}, \tilde{\mathbf{c}})$, and this function is constructed from the stochastic objectives of the initial problem, which, in general, have different characteristics. This fact could call into question the suitability of applying this criterion. As in the minimum risk criterion, in order to fix the probability $\beta \in(0,1)$ for the problem $(\operatorname{SK}(\boldsymbol{\beta}))$, we can consider the possibility of asking the DM to fix a probability for each one of the stochastic objectives, $\beta_{k} \in(0,1)$, and, assuming that the weight vector $\boldsymbol{\mu}$ has a norm equal to one (in any other case, the weights would have to be normalised), we fix the probability $\beta$ for problem $(\operatorname{SK}(\boldsymbol{\beta}))$ as:

$$
\beta=\sum_{k=1}^{q} \mu_{k} \beta_{k}
$$

Now that we have established a possible way to fix the aspiration levels and the probabilities in the two criteria of the maximum probability, in order to state the possible relationships between the efficient sets which are obtained in the two approaches, we consider 
it convenient to indicate that the obtained equivalent deterministic problems (SMR(u)) and $(\operatorname{SK}(\boldsymbol{\beta}))$ are, under certain conditions, reciprocal. Note that from Theorem 2, by simply making $k=1$ and taking optimal solutions instead of efficient solutions, we can affirm that if the distribution function for the random variable $\tilde{f}(\mathbf{x}, \tilde{\mathbf{c}})=\sum_{k=1}^{q} \mu_{k} \tilde{z}_{k}(\mathbf{x}, \tilde{\mathbf{c}})$ is continuous and strictly increasing, it is verified that:

- If $\mathbf{x}^{*}$ is the solution to problem $(\operatorname{SMR}(\mathbf{u}))$ for an aspiration level $u$, then $\left(\mathbf{x}^{* \mathbf{t}}, u\right)^{\mathrm{t}}$ is the solution to problem $(\operatorname{SK}(\boldsymbol{\beta}))$ for a probability level of $\beta=P\left\{\tilde{f}\left(\mathbf{x}^{*}, \tilde{\mathbf{c}}\right) \leq u\right\}$.

- If $\left(\mathbf{x}^{* \mathrm{t}}, u^{*}\right)^{\mathrm{t}}$ is the solution to problem $(\operatorname{SK}(\boldsymbol{\beta}))$ for a probability $\beta$, then $\mathbf{x}^{*}$ is a solution to problem $(\operatorname{SMR}(\mathbf{u}))$ for an aspiration level $u$ such that $\beta=P\left\{\tilde{f}\left(\mathbf{x}^{*}, \tilde{\mathbf{c}}\right) \leq u\right\}$.

The reciprocity between both criteria of maximum probability allows us to carry out a study of the relationships between the problems corresponding to the two different approaches by considering only one of the criteria (either the minimum risk or Kataoka's criteria), and to assert that the same relationships will be verified for the other. From now on, the analysis of the relationships between efficient solutions in the multiobjective and the stochastic approaches by the criteria of maximum probability will be made exclusively for efficiency in probability.

On the other hand, it has to be pointed out that in the two maximum probability criteria the distribution function of the random variables $\tilde{z}_{1}(\mathbf{x}, \tilde{\mathbf{c}}), \ldots, \tilde{z}_{q}(\mathbf{x}, \tilde{\mathbf{c}})$ and $\tilde{f}(\mathbf{x}, \tilde{\mathbf{c}})=\sum_{k=1}^{q} \mu_{k} \tilde{z}_{k}(\mathbf{x}, \tilde{\mathbf{c}})$ are involved. Determining these distribution functions is not an easy issue, because they depend not only on the probability distribution of $\tilde{\mathbf{c}}$, but also on the vector of the decision variables of the problem, $\mathbf{x}$. This leads to the need to establish additional hypotheses regarding the structure of the problem and the distribution of its random parameters. We will focus on linear objective functions under the simple randomness hypothesis, and the hypothesis of vector normality of random parameters, $\tilde{\mathbf{c}}$. Next, we analyse both cases to verify that there is reciprocity between both criteria of maximum probability in both instances. 


\subsubsection{The Linear Simple Randomization Case}

Let us assume that for every $k \in\{1,2, \ldots, q\}$ the objective function $\tilde{z}_{k}(\mathbf{x}, \tilde{\mathbf{c}})$ is linear: $\tilde{z}_{k}(\mathbf{x}, \tilde{\mathbf{c}})=\tilde{\mathbf{c}}_{\mathbf{k}}^{\mathbf{t}} \mathbf{x}$, and that all stochastic objective functions depend on the same random variable, $\tilde{t}$, so that $\tilde{\mathbf{c}}_{\mathbf{k}}=\mathbf{c}_{\mathbf{k}}^{1}+\tilde{t} \mathbf{c}_{\mathbf{k}}^{2}$. Let us also assume that the random variable $\tilde{t}$ is continuous, its expected value $\bar{t}$, and finite variance $v_{t}^{2}$, and its distribution function, $F_{t}$, is strictly increasing. We finally assume that for every $\mathbf{x} \in D$, and for every $k \in\{1,2, \ldots, q\}$, $\mathbf{c}_{\mathbf{k}}^{2 \mathrm{t}} \mathbf{x}>0$.

If these hypotheses are verified, the set of efficient solutions with probabilities $\boldsymbol{\beta}_{1}, \ldots, \boldsymbol{\beta}_{q}$ for the initial stochastic multiobjective problem is the same as the following multiobjective problem:

$$
\operatorname{Min}_{\mathbf{x} \in D}\left(\mathbf{c}_{1}^{1 \mathbf{t}} \mathbf{x}+F_{t}^{-1}\left(\beta_{l}\right) \mathbf{c}_{\mathbf{1}}^{2 \mathbf{t}} \mathbf{x}, \ldots, \mathbf{c}_{\mathbf{q}}^{\mathbf{1 t}} \mathbf{x}+F_{t}^{-1}\left(\beta_{q}\right) \mathbf{c}_{\mathbf{q}}^{\mathbf{2 t}} \mathbf{x}\right)
$$

If the probability fixed for each of the objectives is the same and equal to the probability fixed for the weighted problem, $\beta_{l}=\ldots=\beta_{q}=\beta$, then the set of efficient solutions with probability $\beta$ for the stochastic multiobjective programming problem is the same as for the following problem:

$$
\operatorname{Min}_{\mathbf{x} \in D}\left(\mathbf{c}_{1}^{1 \mathbf{t}} \mathbf{x}+F_{t}^{-1}(\boldsymbol{\beta}) \mathbf{c}_{1}^{2 \mathbf{t}} \mathbf{x}, \ldots, \mathbf{c}_{\mathbf{q}}^{1 \mathbf{t}} \mathbf{x}+F_{t}^{-1}(\boldsymbol{\beta}) \mathbf{c}_{\mathbf{q}}^{2 \mathbf{t}} \mathbf{x}\right)
$$

On the other hand, with the stochastic approach we have:

$$
P(\tilde{f}(\mathbf{x}, \tilde{\mathbf{c}}) \leq u)=P\left(\sum_{k=1}^{q} \boldsymbol{\mu}_{k} \mathbf{c}_{\mathbf{k}}^{\mathbf{1 t}} \mathbf{x}+\tilde{t} \sum_{k=1}^{q} \boldsymbol{\mu}_{k} \mathbf{c}_{\mathbf{k}}^{2 \mathbf{t}} \mathbf{x} \leq u\right)=F_{t}\left(\frac{u-\sum_{k=1}^{q} \boldsymbol{\mu}_{k} \mathbf{c}_{\mathbf{k}}^{\mathbf{1 t}} \mathbf{x}}{\sum_{k=1}^{q} \boldsymbol{\mu}_{k} \mathbf{c}_{\mathbf{k}}^{2 \mathbf{t}} \mathbf{x}}\right)=\beta
$$

which implies that:

$$
u=\sum_{k=1}^{q} \boldsymbol{\mu}_{k} \mathbf{c}_{\mathbf{k}} \mathbf{t} \mathbf{x}+F_{t}^{-1}(\boldsymbol{\beta}) \sum_{k=1}^{q} \boldsymbol{\mu}_{k} \mathbf{c}_{\mathbf{k}}^{2 \mathbf{t}} \mathbf{x}
$$

so, the problem resulting from applying Kataoka's criterion to the weighted problem with probability $\beta$ is: 


$$
\operatorname{Min}_{\mathbf{x} \in D} \sum_{k=1}^{q} \boldsymbol{\mu}_{k} \mathbf{c}_{\mathbf{k}}^{\mathbf{1 t}} \mathbf{x}+F_{t}^{-1}(\boldsymbol{\beta}) \sum_{k=1}^{q} \boldsymbol{\mu}_{k} \mathbf{c}_{\mathbf{k}}^{2 \mathbf{t}} \mathbf{x}
$$

Once the problems for the two criteria are outlined, we can move on and analyse the relationship existing between the optimal solution for problem (2) and the set of efficient solutions with probabilities $\boldsymbol{\beta}_{l}, \ldots, \boldsymbol{\beta}_{q}$.

From this point onwards, if we compare problems (1) and (2), we note that if we apply the weighting method to obtain efficient solutions for problem (1), we obtain problem (2). In other words, in this case, if we weight the stochastic multiobjective programming problem and apply Kataoka's criterion to it for a probability $\beta$, the problem to be solved is the same as if we apply Kataoka's criterion to each objective separately — fixing the same probability for all the stochastic objectives — and apply the weighting method to obtain the efficient solutions for this problem. Therefore, the relationships between these two problems are the same as for any linear multiobjective problem and its associated weighted problem, as described in Theorem 1.

Furthermore, given the reciprocity between the two maximum probability criteria, these relationships are also verified for problem $(\operatorname{SMR}(\mathbf{u}))$, resulting from applying the minimum risk criterion to the weighted problem, and for the set of minimum risk aspiration levels $u_{1}, \ldots, u_{q}$ for the stochastic multiobjective programming problem. Let us explore what happens in the case of normal distribution.

\subsubsection{Linear Objectives with Normal Distribution}

Let us assume that for every $k \in\{1,2, \ldots, q\}$ the objective function $\tilde{z}_{k}(\mathbf{x}, \tilde{\mathbf{c}})$ is linear: $\tilde{z}_{k}(\mathbf{x}, \tilde{\mathbf{c}})=\tilde{\mathbf{c}}_{\mathbf{k}}^{\mathbf{t}} \mathbf{x}$

Let $\widetilde{\mathbf{c}}=\left(\widetilde{\mathbf{c}}_{1}^{\mathbf{t}}, \widetilde{\mathbf{c}}_{2}^{\mathbf{t}}, \ldots, \widetilde{\mathbf{c}}_{\mathbf{q}}^{\mathbf{t}}\right)^{\mathbf{t}}=\left(\widetilde{c}_{11}, \widetilde{c}_{12}, \ldots, \widetilde{c}_{1 n}, \widetilde{c}_{21}, \widetilde{c}_{22}, \ldots, \widetilde{c}_{2 n}, \ldots, \widetilde{c}_{q 1}, \widetilde{c}_{q 2}, \ldots, \widetilde{c}_{q n}\right)^{t}$. Let us also assume that $\widetilde{\mathbf{c}}$ is a random vector multinormal with expected value $\overline{\mathbf{c}}=\left(\overline{\mathbf{c}}_{1}^{\mathbf{t}}, \overline{\mathbf{c}}_{2}^{\mathbf{t}}, \ldots, \overline{\mathbf{c}}_{\mathbf{q}}^{\mathbf{t}}\right)^{\mathbf{t}}=\left(\bar{c}_{11}, \bar{c}_{12}, \ldots, \bar{c}_{1 n}, \bar{c}_{21}, \bar{c}_{22}, \ldots, \bar{c}_{2 n}, \ldots, \bar{c}_{q 1}, \bar{c}_{q 2}, \ldots, \bar{c}_{q n}\right)^{\mathrm{t}}$ and variance and positive definite covariance matrix $\mathbf{V}$ : 


$\mathbf{V}=\left(\begin{array}{c|c|c|c|c|c}\mathbf{V}_{1} & \mathbf{V}_{12} & \ldots & \mathbf{V}_{1 \mathrm{~s}} & \ldots & \mathbf{V}_{1 \mathrm{q}} \\ \hline \mathbf{V}_{21} & \mathbf{V}_{2} & \ldots & \mathbf{V}_{2 \mathrm{~s}} & \ldots & \mathbf{V}_{2 \mathrm{q}} \\ \hline \ldots & \ldots & \ldots & \ldots & \ldots & \ldots \\ \hline \mathbf{V}_{\mathrm{k} 1} & \mathbf{V}_{\mathrm{k} 2} & \ldots & \mathbf{V}_{\mathrm{ks}} & \ldots & \mathbf{V}_{\mathbf{k q}} \\ \hline \ldots & \ldots & \ldots & \ldots & \ldots & \ldots \\ \hline \mathbf{V}_{\mathbf{q} 1} & \mathbf{V}_{\mathrm{q} 2} & \ldots & \mathbf{V}_{\mathrm{qs}} & \ldots & \mathbf{V}_{\mathrm{q}}\end{array}\right)$

Let us assume also that $\mathbf{0} \notin D$.

If these hypotheses are verified, the probability of the random variable $\tilde{f}(\mathbf{x}, \widetilde{\mathbf{c}})=\sum_{k=1}^{q} \boldsymbol{\mu}_{k} \widetilde{\mathbf{c}}_{\mathbf{k}}^{\mathbf{t}} \mathbf{x}$ being less or equal to $u$ is:

$$
P\left\{\sum_{k=1}^{q} \boldsymbol{\mu}_{k} \widetilde{\mathbf{c}}_{\mathbf{k}}^{\mathbf{t}} \mathbf{x} \leq u\right\}=\Phi\left(\frac{u-\sum_{k=1}^{q} \boldsymbol{\mu}_{k} \overline{\mathbf{c}}_{\mathbf{k}}^{\mathbf{t}} \mathbf{x}}{\sqrt{\sum_{k=1}^{q} \boldsymbol{\mu}_{k}^{2} \mathbf{x}^{\mathbf{t}} \mathbf{V}_{\mathbf{k}} \mathbf{x}+2 \sum_{\substack{k, s=1 \\ k<s}}^{q} \boldsymbol{\mu}_{k} \boldsymbol{\mu}_{s} \mathbf{x}^{\mathbf{t}} \mathbf{V}_{\mathbf{k s}} \mathbf{x}}}\right)=\beta
$$

where $\Phi$ is the distribution function of the standard normal distribution. Then:

$$
u=\sum_{k=1}^{q} \boldsymbol{\mu}_{k} \overline{\mathbf{c}}_{\mathbf{k}}^{\mathbf{t}} \mathbf{x}+\Phi^{-1}(\boldsymbol{\beta}) \sqrt{\sum_{k=1}^{q} \boldsymbol{\mu}_{k}^{2} \mathbf{x}^{\mathbf{t}} \mathbf{V}_{\mathbf{k}} \mathbf{x}+2 \sum_{\substack{k, s=1 \\ k<s}}^{q} \boldsymbol{\mu}_{k} \boldsymbol{\mu}_{s} \mathbf{x}^{\mathbf{t}} \mathbf{V}_{\mathbf{k s}} \mathbf{x}}
$$

and, the solution to the weighted problem (S), using Kataoka's criterion, is given by the solution to the problem:

$$
\operatorname{Min}_{\mathbf{x} \in D} \sum_{k=1}^{q} \boldsymbol{\mu}_{k} \overline{\mathbf{c}}_{\mathbf{k}}^{\mathbf{t}} \mathbf{x}+\Phi^{-1}(\boldsymbol{\beta}) \sqrt{\sum_{k=1}^{q} \boldsymbol{\mu}_{k}^{2} \mathbf{x}^{\mathbf{t}} \mathbf{V}_{\mathbf{k}} \mathbf{x}+2 \sum_{\substack{k, s=1 \\ k<s}}^{q} \boldsymbol{\mu}_{k} \boldsymbol{\mu}_{s} \mathbf{x}^{\mathbf{t}} \mathbf{V}_{\mathbf{k s}} \mathbf{x}}
$$

Given that the normal distribution function is strictly increasing and continuous, there still is reciprocity between the optimal solutions for the problem obtained by applying the minimum risk criterion to the weighted problem, and for problem (3) obtained by applying Kataoka's criterion.

Note that problem (3) can also be expressed as: 


$$
\operatorname{Min}_{\mathbf{x} \in D} \sum_{k=1}^{q} \mu_{k} \bar{z}_{k}(\mathbf{x})+\alpha \sigma(\mathbf{x})
$$

with $\bar{z}_{k}(\mathbf{x})=\overline{\mathbf{c}}_{\mathbf{k}}^{\mathbf{t}} \mathbf{x}, \sigma(\mathbf{x})=\sqrt{\sum_{k=1}^{q} \boldsymbol{\mu}_{k}^{2} \mathbf{x}^{\mathbf{t}} \mathbf{V}_{\mathbf{k}} \mathbf{x}+2 \sum_{\substack{k, s=1 \\ k<s}}^{q} \boldsymbol{\mu}_{k} \boldsymbol{\mu}_{s} \mathbf{x}^{\mathbf{t}} \mathbf{V}_{\mathbf{k s}} \mathbf{x}}$ and $\alpha=\Phi^{-1}(\beta)$.

Once problem (4) has been expressed, we consider again the possible existence of a relationship between its optimal solutions and some of the concepts of efficient solution for stochastic multiobjective programming problems. We will relate the optimal solution for problem (4) to the expected value standard deviation efficient solution. The following proposition relates the optimal solution for problem (4), obtained by resolving the weighted problem using Kataoka's criterion, with the set $\mathcal{E}_{\mathrm{E} \sigma}$.

\section{Proposition 1}

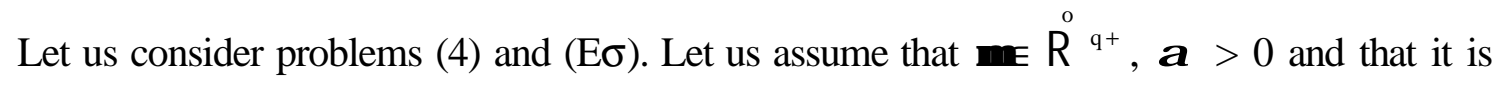
verified that $\sigma_{k s}(\mathbf{x})=0$ for every $k, s \in\{1,2, \ldots, q\}$, with $k \neq s$ and for every $\mathbf{x} \in D$. Then, if $\mathbf{x}^{*}$ is the optimal solution for (4), it is also the efficient solution for (E $\left.\sigma\right)$.

\section{Demonstration}

The demonstration is done by reductio ad absurdum.

Let $\mathbf{x}^{*} \in D$ be the optimal solution for (4) and let us assume that it is not the efficient solution for $(\mathrm{E} \sigma)$. In this case, there is a solution $\mathbf{x}^{\prime}$ that dominates $\mathbf{x}$, and so, for every $k \in\{1,2, \ldots$, $q\}$ :

$$
\bar{z}_{k}\left(\mathbf{x}^{\prime}\right) \leq \bar{z}_{k}\left(\mathbf{x}^{*}\right) \text { and } \boldsymbol{\sigma}_{k}\left(\mathbf{x}^{\prime}\right) \leq \boldsymbol{\sigma}_{k}\left(\mathbf{x}^{*}\right)
$$

and there is at least a $s \in\{1,2, \ldots, q\}$ for which the inequality is strict, that is:

$$
\bar{z}_{s}\left(\mathbf{x}^{\prime}\right)<\bar{z}_{s}\left(\mathbf{x}^{*}\right) \text { or } \boldsymbol{\sigma}_{s}\left(\mathbf{x}^{\prime}\right)<\boldsymbol{\sigma}_{s}\left(\mathbf{x}^{*}\right)
$$

Given that $\boldsymbol{\mu} \in \mathrm{R}^{\mathrm{o}} \mathrm{q}^{+}$, for every $k \in\{1,2, \ldots, q\}$ it is verified that:

$$
\mu_{k} \bar{z}_{k}\left(\mathbf{x}^{\prime}\right) \leq \mu_{k} \bar{z}_{k}\left(\mathbf{x}^{*}\right)
$$




$$
\mu_{k} \sigma_{k}\left(\mathbf{x}^{\prime}\right) \leq \mu_{k} \sigma_{k}\left(\mathbf{x}^{*}\right)
$$

and there is at least a $s \in\{1,2, \ldots, q\}$ for which:

$$
\mu_{s} \bar{z}_{s}\left(\mathbf{x}^{\prime}\right)<\mu_{s} \bar{z}_{s}\left(\mathbf{x}^{*}\right) \text { or } \mu_{s} \sigma_{s}\left(\mathbf{x}^{\prime}\right)<\mu_{s} \sigma_{s}\left(\mathbf{x}^{*}\right)
$$

Given that the variance is always non-negative, if we raise both sides of the inequality (6) to the power of 2 , we obtain:

$$
\mu_{k}^{2} \sigma_{k}^{2}\left(\mathbf{x}^{\prime}\right) \leq \mu_{k}^{2} \sigma_{k}^{2}\left(\mathbf{x}^{*}\right)
$$

Adding up (5) and (7) in $k$ we obtain:

$$
\begin{gathered}
\sum_{k=1}^{q} \boldsymbol{\mu}_{k} \bar{z}_{k}\left(\mathbf{x}^{\prime}\right) \leq \sum_{k=1}^{q} \boldsymbol{\mu}_{k} \bar{z}_{k}\left(\mathbf{x}^{*}\right) \\
\sum_{k=1}^{q} \mu_{k}^{2} \boldsymbol{\sigma}_{\mathrm{k}}^{2}\left(\mathbf{x}^{\prime}\right) \leq \sum_{k=1}^{q} \mu_{k}^{2} \boldsymbol{\sigma}_{\mathrm{k}}^{2}\left(\mathbf{x}^{*}\right)
\end{gathered}
$$

From (9) we deduce that:

$$
\boldsymbol{\alpha} \sqrt{\sum_{k=1}^{q} \mu_{k}^{2} \sigma_{\mathrm{k}}^{2}\left(\mathbf{x}^{\prime}\right)} \leq \boldsymbol{\alpha} \sqrt{\sum_{k=1}^{q} \mu_{k}^{2} \boldsymbol{\sigma}_{\mathrm{k}}^{2}\left(\mathbf{x}^{*}\right)}
$$

and that one of the inequalities, either (8) or (10) is strict.

From the above expressions we obtain:

$$
\sum_{k=1}^{q} \boldsymbol{\mu}_{k} \bar{z}_{k}\left(\mathbf{x}^{\prime}\right)+\boldsymbol{\alpha} \sqrt{\sum_{k=1}^{q} \mu_{k}^{2} \boldsymbol{\sigma}_{k}^{2}\left(\mathbf{x}^{\prime}\right)}<\sum_{k=1}^{q} \boldsymbol{\mu}_{k} \bar{z}_{k}\left(\mathbf{x}^{*}\right)+\boldsymbol{\alpha} \sqrt{\sum_{k=1}^{q} \mu_{k}^{2} \boldsymbol{\sigma}_{k}^{2}\left(\mathbf{x}^{*}\right)}
$$

and this is contrary to $\mathbf{x}^{*}$ being the optimal solution for (4).

Therefore, this proposition asserts that if the covariances of the stochastic objective functions of the problem are zero, the optimal solution obtained by applying Kataoka's criterion on the weighted problem, with strictly positive weights, is an expected value standard deviation efficient solution for the stochastic multiobjective problem, if the value of the 
parameter $\alpha$ is strictly positive. Given that $\alpha=\Phi^{-1}(\beta)$, if the fixed probability $\beta$ is greater than 0.5 , then $\alpha>0$. In any other case, the optimal solution to problem (4) does not have to be the expected value standard deviation as shown in the example below.

Finally, given that in this case there is also reciprocity between the optimal solution for the problem (4) and for the problem obtained by applying the minimum risk criterion to the weighted problem, the results obtained for Kataoka's criterion can be extrapolated to the minimum risk criterion.

\section{Example}

Let us consider the following stochastic bi-objective programming problem:

$$
\begin{array}{cl}
\operatorname{Min}_{\mathbf{x}} & \left(\tilde{c}_{11} x_{1}+\tilde{c}_{12} x_{2}, \tilde{c}_{21} x_{1}+\tilde{c}_{22} x_{2}\right) \\
\text { s.t } & x_{1}+2 x_{2} \geq 4 \\
& x_{1}, x_{2} \leq 3 \\
& x_{1}, x_{2} \geq 0
\end{array}
$$

with $\tilde{\mathbf{c}}=\left(\tilde{c}_{11}, \tilde{c}_{12}, \tilde{c}_{21}, \tilde{c}_{22}\right)^{\mathrm{t}}$ being a random vector multinormal with expected value $\overline{\mathbf{c}}=(0.5,1,1,2.5)^{\mathrm{t}}$ and with positive definite covariance matrix :

$$
\mathbf{V}=\left(\begin{array}{cccc}
25 & 0 & 0 & 3 \\
0 & 25 & 3 & 0 \\
0 & 3 & 1 & 0 \\
3 & 0 & 0 & 9
\end{array}\right)
$$

In order to illustrate some of the results obtained in this work, we are going to solve this problem using some of the criteria previously considered. Before carrying on, it should be noted that given that the feasible set for this problem is a non-empty, closed and bounded set (see Figure 1) and, given that the objective functions for the equivalent deterministic problems we will obtain are continuous in this feasible set, we can assert the existence of either optimal or efficient solutions (depending on the case) for them.

We now apply the minimum variance, expected value standard deviation, and Kataoka's criteria. 
a) Minimum variance criterion: From the data of the problem, the set of minimum variance efficient solutions is the same as for problem:

$$
\begin{gathered}
\operatorname{Min}_{\mathbf{x}}\left(25 x_{1}^{2}+25 x_{2}^{2}, x_{1}^{2}+9 x_{2}^{2}\right) \\
\text { s.t } x_{1}+2 x_{2} \geq 4 \\
x_{1}, x_{2} \leq 3 \\
x_{1}, x_{2} \geq 0
\end{gathered}
$$

which includes the variances of the stochastic objectives. This set is the segment that joins point $\mathbf{A}$, with coordinates $(0.8,1.6)$, to point $\mathbf{B}$, with coordinates $(2.769231,0.6153846)$, in Figure 1.

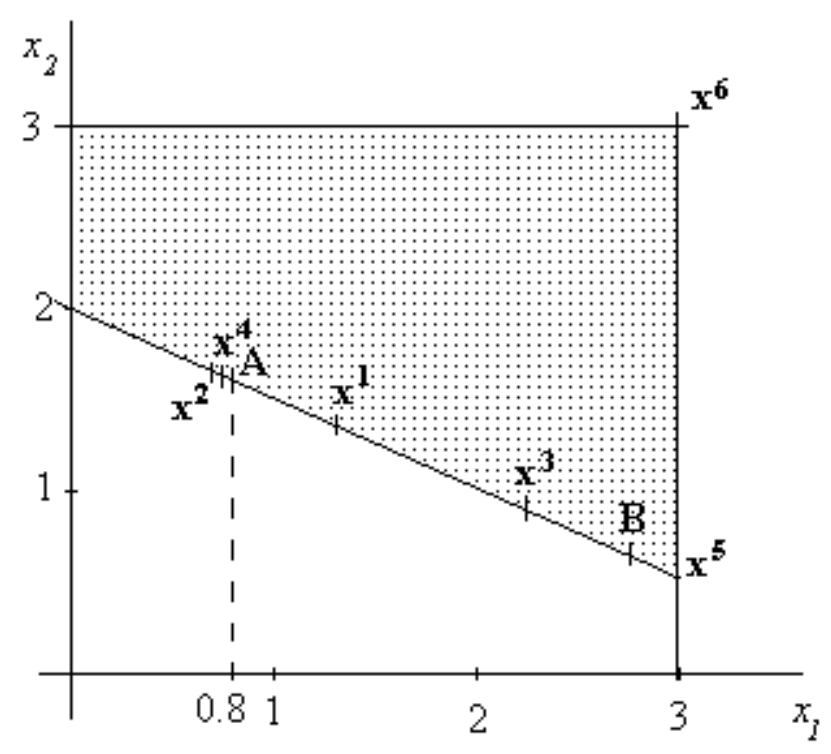

Figure 1

On the other hand, if we solve the previous problem by using the stochastic approach, weighting the stochastic objectives with weights $\mu_{1}$ and $\mu_{2}$ and then applying the minimum variance criterion, we obtain the problem:

$$
\begin{array}{cc}
\operatorname{Min}_{\mathbf{x}} & \mu_{1}^{2}\left(25 x_{1}^{2}+25 x_{2}^{2}\right)+\mu_{2}^{2}\left(x_{1}^{2}+9 x_{2}^{2}\right)+2 \mu_{1} \mu_{2}\left(6 x_{1} x_{2}\right) \\
\text { s.t } & x_{1}+2 x_{2} \geq 4 \\
& x_{1}, x_{2} \leq 3 \\
& x_{1}, x_{2} \geq 0
\end{array}
$$


If the following weights $\mu_{1}=0.3, \mu_{2}=0.7$ are used in this problem, we obtain the solution $\mathbf{x}^{1}=(1.316375,1.341812)^{\mathrm{t}}$, which is a minimum variance solution, but, if we fix the weights $\mu_{1}=0.75, \mu_{2}=0.35$, the solution we obtain is $\mathbf{x}^{2}=(0.7302074,1.634896)^{\mathrm{t}}$, which is a minimum variance dominated solution.

This example shows that if the covariances of the stochastic objectives are not zero, the minimum variance solutions to the weighted problem do not have to be minimum variance efficient, as pointed out in section 3 .

\section{b) Kataoka's criterion and expected value standard deviation efficiency.}

Let us now consider solving the weighted problem by apply Kataoka's criterion. The resulting problem, for a probability $\beta$ is:

$$
\begin{array}{cc}
\operatorname{Min}_{\mathrm{x}} \mu_{l}\left(0.5 x_{1}+x_{2}\right) & +\mu_{2}\left(x_{1}+2.5 x_{2}\right)+\Phi^{-1}(\beta) \sqrt{\mu_{1}^{2}\left(25 x_{1}^{2}+25 x_{2}^{2}\right)+\mu_{2}^{2}\left(x_{1}^{2}+9 x_{2}^{2}\right)+12 \mu_{l} \mu_{2} x_{1} x_{2}} \\
\text { s.t } & x_{1}+2 x_{2} \geq 4 \\
& x_{1}, x_{2} \leq 3 \\
& x_{1}, x_{2} \geq 0
\end{array}
$$

As we have seen in section 3, if the covariance of the stochastic objective functions is zero and the probability fixed is greater than 0.5 , for each vector of weights $\boldsymbol{\mu}>\mathbf{0}$ the optimal solution for problem (12) is expected value standard deviation efficient; but if either of these two hypotheses is not verified, the previous statement is not necessarily true, as we show next. First, we will relax the first hypothesis and then the second one.

To begin with, it has to be noted that the set of expected value standard deviation efficient solutions for the initial problem is the segment that joins points $\mathbf{A}$ and $\mathbf{x}^{\mathbf{5}}$ in Figure 1.

Let us consider problem (12) for a probability $\beta=0.95$. If the weight vector is fixed at $\boldsymbol{\mu}=(0.2,0.8)^{\mathrm{t}}$, we obtain the solution $\mathbf{x}^{3}=(2.255366,0.8723169)$, which belongs to the expected value standard deviation efficient set. However, for the weight vector $\boldsymbol{\mu}=(0.7,0.3)$, the solution to the weighted problem is $\mathbf{x}^{4}=(0.7559553,1.622022)$. As shown in the graph, this solution does not belong to the expected value standard deviation efficient set. 
On the other hand, let us assume that the covariance of the stochastic objective functions is zero and that they have a normal distribution; that is, we assume that the vector $\tilde{\mathbf{c}}=\left(\tilde{c}_{11}, \tilde{c}_{12}, \tilde{c}_{21}, \tilde{c}_{22}\right)^{\mathrm{t}}$ is a random vector multinormal with expected value $\overline{\mathbf{c}}=(0.5,1,1,2.5)^{\mathrm{t}}$ and positive definite covariance matrix, $\mathbf{V}$ :

$$
\mathbf{V}=\left(\begin{array}{cccc}
25 & 0 & 0 & 0 \\
0 & 25 & 0 & 0 \\
0 & 0 & 1 & 0 \\
0 & 0 & 0 & 9
\end{array}\right)
$$

The set of expected value standard deviation efficient solutions for this problem is the same as for the previous one, but the problem obtained from applying Kataoka's criterion to the weighted problem for a probability $\beta$ is now:

$$
\begin{aligned}
& \operatorname{Min}_{\mathbf{x}} \mu_{1}\left(0.5 x_{1}+x_{2}\right)+\mu_{2}\left(x_{1}+2.5 x_{2}\right)+\Phi^{-1}(\beta) \sqrt{\mu_{1}^{2}\left(25 x_{1}^{2}+25 x_{2}^{2}\right)+\mu_{2}^{2}\left(x_{1}^{2}+9 x_{2}^{2}\right)} \\
& \text { s.t } \quad x_{1}+2 x_{2} \geq 4 \\
& x_{1}, x_{2} \leq 3 \\
& x_{1}, x_{2} \geq 0
\end{aligned}
$$

As previously shown, for any weight vector, the solutions for this problem are expected value standard deviation efficient solutions, if the probability fixed is greater than 0.5 , but this does not necessarily have to be the case if the probability fixed is less than 0.5. Thus, for a weight vector $\boldsymbol{\mu}=(0.8,0.2)^{\mathrm{t}}$, if the probability fixed is $\beta=0.4$, the solution obtained is $\mathbf{x}^{5}=(3,0.5)$; but if the probability fixed is $\beta=0.2$, the solution obtained is $\mathbf{x}^{6}=(3,3)$, which is not expected value standard deviation efficient solution, as shown in Figure 1.

\section{Conclusions}

In this paper, it is shown that the achievement of efficient solutions in problems of stochastic multiobjective programming is carried out by combining the techniques of both stochastic programming and multiobjective programming, in a double transformation of the problem. The question we study is if the order in which the transformations are carried out has an influence in the set of efficient solutions, which are obtained. Thus, we pretend to determine 
which approach is most appropriate for the achievement of efficient solutions of stochastic multiobjective programming problems.

Our study shows that, when among the stochastic objectives there exists a stochastic dependence, the order of transformations can lead to an efficient solution by following a certain order which is a non-efficient solution according to the inverse order.

From this work, we can conclude that the achievement of efficient solutions of stochastic multiobjective programming problems using the stochastic approach, by using the weighted method, is closely linked to problem resolution using the multiobjective approach.

Specifically, the results obtained with the minimum variance criterion and the maximum probability criteria establish that, in order to have a relationship between the optimal solutions for the weighted problem obtained with these criteria, and some of the concepts of efficient solutions defined in the multiobjective approach, it is necessary for the covariances of the objectives to be equal to zero in some cases.

This suggests to some extent that the multiobjective approach "forgets" the possible existence of stochastic dependencies between objectives. Note that the main criticism of the application of the multiobjective approach is that, when obtaining the equivalent deterministic problem, a transformation criterion is applied to each objective function separately. This means that in the resolution of the stochastic multiobjective programming problem, the possible stochastic dependency between the objectives is not taken into account.

The stochastic approach takes into account the dependency between them, even if only partially, in terms of covariance.

In this sense, given that in real situations it can be frequent that there exist stochastic dependences among objectives, we can assert that when these dependences exist, the stochastic approach is more appropriate for the achievement of efficient solutions than the multiobjective approach because it reflects to a certain extent the stochastic and multiobjective nature of the real problem. 


\section{References}

Ben Abdelaziz, F., 1992. L'efficacité en Programmation Multi-objectifs Stochastique. Ph. D. Thesis, Université de Laval, Québec.

Ben Abdelaziz, F., Lang, P., Nadeau, R., 1997. Distributional Unanimity Multiobjective Stochastic Linear Programming. In: Climaco, J. (Ed.: Multicriteria Analysis: Proceedings of the With Conference on MCDM, pp. 225-236. Springer-Verlag.

Ben Abdelaziz, F., Lang, P., Nadeau, R., 1999. Dominance and Efficiency in Multicriteria Decision under Uncertainty. Theory and Decision, 47, 191-211.

Caballero, C., Cerdá, E., Muñoz, M.M., Rey, L., 2000. Relations among Several Efficiency Concepts in Stochastic Multiple Objective Programming. Research and Practice in Multiple Criteria Decision Making, Edited by Y. Y. Haimes and R. Steuer, Lectures Notes in Economics and Mathematical Systems, Springer-Verlag, Berlin, Germany, Vol. 487, 57-68.

Caballero, R., Cerdá, E., Muñoz, M.M., Rey, L., Stancu Minasian, I. M., (2001), Efficient Solution Concepts and Their Relations in Stochastic Multiobjective Programming. Journal of Optimization, Theory and Applications, Vol. 110, 1, 53-74.

Chankong, V., Haimes, Y.Y., 1983. Multiobjective Decision Making: Theory and Methodology. North-Holland, New York.

Goicoechea, A., Hansen, D. R., Duckstein, L., 1982. Multiobjective Decision Analysis with Engineering and Business Applications. John Wiley and Sons, New York.

Hogg, R. V., Craig, A. T., 1989. Introduction to Mathematical Statistics. MacMillan Publishing Co., New York.

Kall, P., Wallace, S.W., 1994. Stochastic Programming. John Wiley and sons, Chichester.

Liu, B., Iwamura, K., 1997. Modelling Stochastic Decision Systems Using DependentChance Programming. European Journal of Operational Research, 101, 193-203.

Prékopa, A., 1995. Stochastic Programming. Kluwer Academic Publishers. Dordrecht.

Sawaragi, Y., Nakayama H., Tanino T., 1985. Theory of Multiobjective Optimization. Academic Press, New York.

Slowinski, R., Teghem, J. (Ed.), 1990. Stochastic Versus Fuzzy Approaches to Multiobjective Mathematical Programming Under Uncertainty. Kluwer Academic Publishers, Dordrecht.

Stancu-Minasian, I. M., 1984. Stochastic Programming with Multiple Objective Functions. D. Reidel Publishing Company, Dordrecht.

Stancu-Minasian, I., Tigan, S., 1984. The Vectorial Minimum Risk Problem. Proceedings of the Colloquium on Approximation and Optimization. Cluj-Napoca, 321-328. 
White, D. J., 1982. Optimality and Efficiency. John Wiley and Sons, Chichester. 